\title{
Hearing of Old World Monkeys (Cercopithecinae)'
}

\author{
WILLIAM C. STEBBINS \\ Kresge Hearing Research Institute and Departments of Otorhinolaryngology \\ and Psychology, The University of Michigan, \\ Ann Arbor, Michigan 48104
}

\begin{abstract}
The characteristics of normal hearing were examined in the laboratory for seven species of Old World monkeys. Operant conditioning procedures, coupled with standard audiometric testing methods, were used to assess thresholds of hearing, frequency range of hearing, and differential sensitivity to auditory intensity and frequency. To produce tonal stimulation, an animal was trained to touch and maintain manual contact with a contact-sensitive key and to report hearing the tone by lifting his hand from the key; this response was followed by food reinforcement. When the reporting response occurred without the auditory signal, the animal was punished by a short suspension from the experiment. Additional contingencies were added to ensure stable and reliable responding, and threshold and differential acuity determinations were then made. Threshold was defined as the stimulus value responded to correctly $50 \%$ of the time. The frequency range of hearing of all the cercopithecoids tested extended from 60 to $40,000 \mathrm{~Hz}$, an octave above the upper bound of $20,000 \mathrm{~Hz}$ for man but well below the $60-70,000 \mathrm{~Hz}$ limit for some prosimians. Absolute sensitivity for tonal stimulation in the most sensitive frequency range $(1-8 \mathrm{kHz})$ was about $2 \times 10^{-4}$ microbars, comparable to that of other primates tested, including man. Thus, the Old World monkey appears only slightly less sensitive than man to small changes in intensity and frequency of acoustic stimulation. At $1000 \mathrm{~Hz}$ at $60 \mathrm{~dB}$ above the threshold of audibility, his limit of resolution is about $5 \mathrm{~Hz}$ for frequency and $2 \mathrm{~dB}$ for intensity.
\end{abstract}

Hearing has not been one of the distinguishing features traditionally included as part of the definition of the order Primates and yet hearing serves them in a variety of functions important, if not always critical, to their daily existence. Intraspecies communication and the detection of prey and predator are perhaps two of the most widely used of these functions. The emphasis for criteria in defining this mammalian order has always rested on a contra-distinction between the dramatic evolutionary development of their visual apparatus and the equally spectacular reduction in their offactory equipment and sensitivity. If audition is ever mentioned in discussions of primate taxonomy, it is usually briefly dispatched with a short discussion of external and middle ear morphology. There is a marked lack of concern with how primates use these structures in hearing. Even very recently, Napier and Napier ('67) discuss the use of the external ear in primates as a device for temperature regulation and in visual communication. About hearing we are told only that there is an "auditory function" (Napier and Napier, '67: 20); no further details are given.

To a considerable extent, this lack of attention to the auditory capabilities of primates has reflected man's ignorance of them. In the past few years, the situation has changed, and we now have reliable audiometric data obtained under laboratory conditions from representatives of at least nine primate families (Stebbins, ' 71 ). On the basis of their frequency range of hearing and sensitivity, the primates divide conveniently into two groups - the prosimians, resembling the other mammals in their ability to hear high frequencies (to $70 \mathrm{kHz}$ ) and with their somewhat reduced

1 The research is supported by grants NS 05077 and NS 05785 from the National Institute of Neurological Diseases and Stroke. 
sensitivity to low and midrange frequencies (below $8 \mathrm{kHz}$ ); and the Anthropoidea, limited to frequencies below $40 \mathrm{kHz}$ with somewhat enhanced sensitivity around $1 \mathrm{kHz}$. However, even these generalizations have to be considered tentative since the number of species tested is less than 20 .

In this paper, the previous review (Stebbins, '71) has been updated and our findings on seven species ( 4 genera) of the Cercopithecinae subfamily of Old World monkeys (Cercopithecidae) are presented: Macaca arctoides, $M$. fascicularis, $M$. mulatta, M. nemestrina, Cercopithecus aethiops, Erythrocebus patas, and Papio papio. The differences between species are no greater than the intraspecies differences on the audiometric measures we have taken, so that we are able to present our findings for the subfamily Cercopithecinae as a whole. These findings include auditory thresholds for pure tones ranging from 60 $\mathrm{Hz}$ to $40 \mathrm{kHz}$ in addition to frequency difference and intensity difference thresholds. The difference thresholds, however, are limited to two genera: Macaca and Papio. Such information is not closely related to the natural acoustic environment of the monkey; rather, it gives us an estimate of the lowest sound pressure to which his ear is sensitive and of the resolving power of his ear along the dimensions of wavelength and amplitude (intensity or sound pressure). The Old World monkey is probably the most popular choice for field and laboratory study and it behooves us to know something about his hearing acuity. Before we can interpret the significance of his hearing within the context of his natural environment, we must first specify the characteristics of his ear as an analyzer of sound. The remainder of this paper will be devoted to a description of the methods for the behavioral measurement of hearing in nonhuman primates and a discussion of the findings for the Cercopithecinae and their relation to other primate families.

\section{METHOD}

Most of our animals are juvenile and subadult males (probable age range between 3 and 7 years). The majority have been obtained from importers, although two taxa (baboons and vervets) were born and raised in this country. Upon arrival in the laboratory, the monkeys undergo a sixweek quarantine period which includes a series of TB tests and a general health examination. Subsequently they are fitted with collar and leash and trained to leave their cages to enter special restraint chairs (fig. 1). They are then placed in a soundtreated room where the behavioral conditioning and hearing testing procedures are carried out. Earphones are carefully fitted over each ear and a special tube and chute are arranged to deliver small whole-diet pellets directly to the monkey's mouth (see fig. 1). The complete details of the equipment and of the training and testing procedures have been described elsewhere (Moody, Stebbins and Miller, '70; Stebbins, '70; Stebbins and Coombs, '72).

The monkey is required to make and maintain manual contact with a metal disc located on an extended arm in front of the chair (see fig. 1) until presented with a pure tone. Lifting his hand from the disc (breaking contact) while the tone is on indicates a correct detection and is immediately followed by a food pellet. Failure to break contact with the disc during the tone counts as a failure to hear and has no programmed consequences. Removal of his hand from the disc when the tone is not present produces a six-second time-out from the experiment which is indicated by the extinction of a red light directly in front of the animal. During that period, the experiment is temporarily interrupted and further experimental events (including delivery of food) are postponed. The timeout acts as an effective punishment for guessing, but is without the emotional disturbance so of ten associated with electric shock.

We consider maintained contact with the metal disc an observing response which places the animal in an effective listening position such that he is able to respond quickly to the tone simply by lifting his hand. A two and one-half second "trial" occurs randomly between one and nine seconds after the initiation of a contact response. On $67 \%$ of the trials, a tone is actually presented and the animal is reinforced with food if he removes his hand from the disc before the tone is turned off. The remaining $33 \%$ of the trials are considered "catch trials" - no tone is pre- 


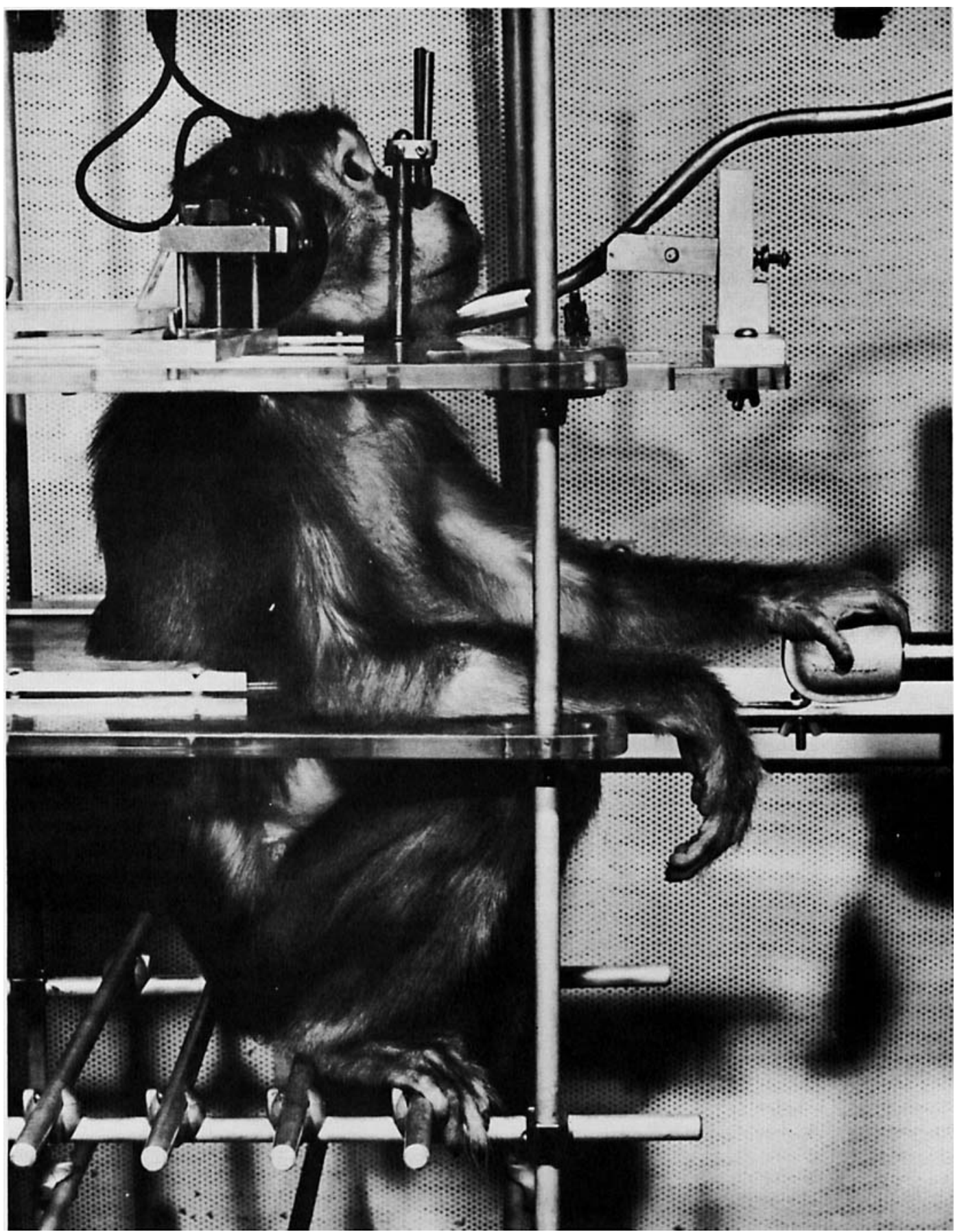

Fig. I Subject (Macaca nemestrina) seated in the restraining chair for hearing testing. Earphones, response disc, and feeder tube are shown. The monkey is in listening position with his hand on the response disc prior to tone stimulation. 
sented and lifting his hand from the disc at this time is punished by a time-out. These silent trials are used to monitor the animal's guess rate. We reject the data if the responses to these catch trials exceed $10 \%$ of the total number presented. A trained animal rarely responds to these trials and almost always reports the tone correctly.

The picture of a trained animal is serene and relatively uninteresting. He sits very quietly in his chair with his hand or forearm draped over the response disc (see fig. 1). Upon hearing a tone, he lifts his arm a few inches from the key and immediately replaces it, at the same time extending his tongue to receive the food pellet from the delivery chute.

When the behavior has become stable under these conditions, threshold testing for pure tones is begun. A variant of Békésy audiometry (tracking, up-down, or titration) is used. For each frequency, the tone is presented initially well above the estimated threshold for the animal. Following each correct report of the stimulus, its intensity when next presented is decreased by $5-10 \mathrm{~dB}$; when the subject fails to report the stimulus, its intensity is increased subsequently by the same amount on the next trial. In the traditional manner, threshold is defined as the value of stimulation correctly detected in one-half of the trials in which it was presented.

Essentially the same procedure is used in the determination of frequency and in- tensity difference thresholds. For the former, a pure tone is presented repetitively; maintained contact with the disc is followed by a second pure tone of different frequency alternating with the first or standard for two and one-half seconds. The monkey is reinforced with food for correctly discriminating the frequency difference by breaking contact with the disc. Correct responses serve to reduce the frequency difference between the two tones; failure to break contact with the disc when the two tones are alternating serves to increase the frequency difference between them on the next trial. Difference thresholds are determined for frequency and in a very similar manner for tonal intensity.

In figure 2 , the tracking procedure is illustrated in diagrammatic form. The conditions are those for determination of the frequency difference threshold; however, the basic principle of the tracking procedure - that of adjustment of the stimulus difference by the subject's response - is the same regardless of the situation in which it is used. The standard stimulus is a $1-\mathrm{kHz}$ tone and the second or variable stimulus is at an initial value $24 \mathrm{~Hz}$ from the standard. In a brief warmup, the animal responds correctly to the stimulus difference, thus gradually reducing that difference to about $9 \mathrm{~Hz}$, which is calculated as the average frequency difference threshold at $1 \mathrm{kHz}$ and is based on the data collected from trial 10 through trial 29 (see

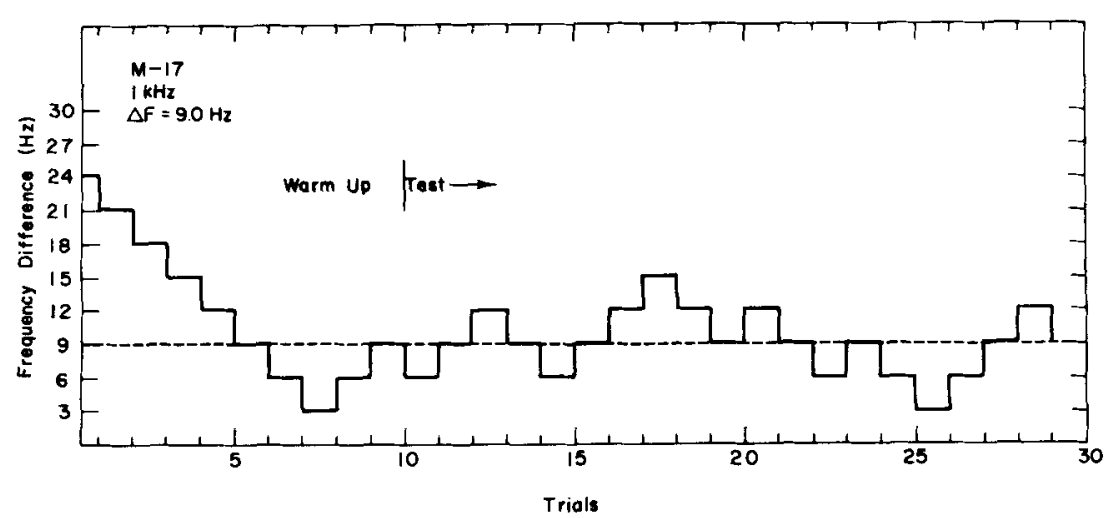

Fig. 2 Use of the tracking method in the determination of frequency difference thresholds. The session begins after a brief "warmup" at the point labeled "test" on the figure. Correct reports reduce the difference between the frequencies; following a failure to report, the difference is increased. The calculated threshold is indicated by the dashed horizontal line at $9 \mathrm{~Hz}$. 


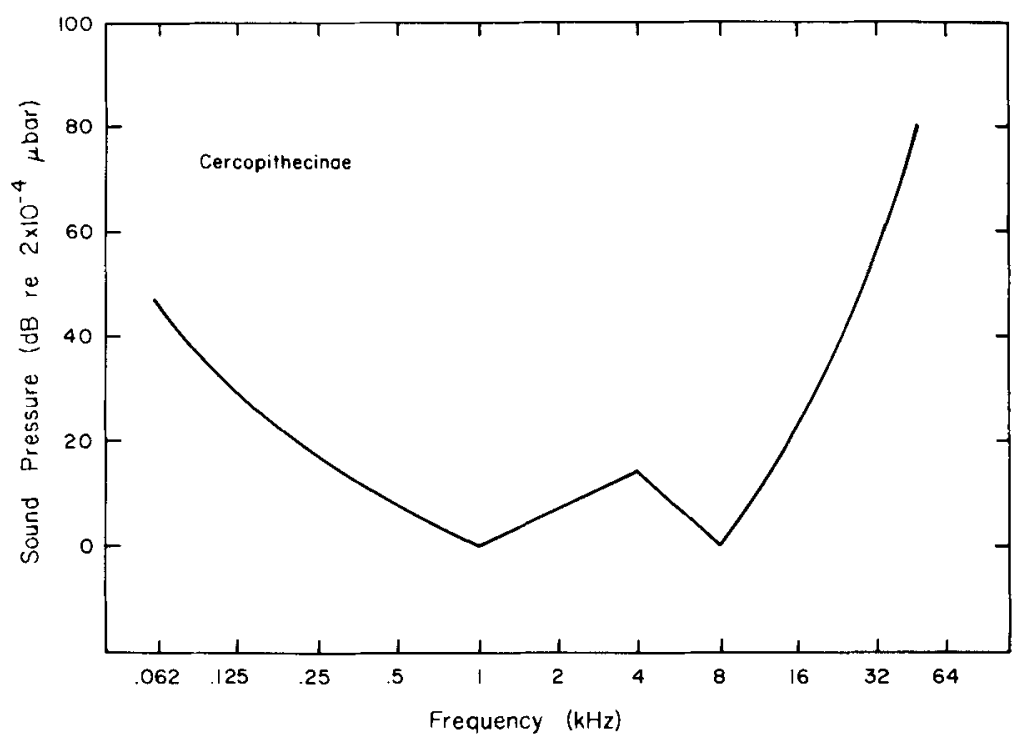

Fig. 3 Threshold of hearing function from $60 \mathrm{~Hz}$ to $45 \mathrm{kHz}$ for Cercopithecinae based on data obtained from four genera.

fig. 2). Thresholds are determined in this manner over a wide range of standard frequencies.

\section{RESULTS AND DISCUSSION}

A representative hearing function for pure tones for the cercopithecine monkeys is shown in figure 3. Although individual animals may differ slightly, most of the essential features of the function are evident for all animals:

1. The frequency range extends from below $60 \mathrm{~Hz}$ to as high as $40-45 \mathrm{kHz}$.

2. Maximum sensitivity is in the midrange frequencies between $1 \mathrm{kHz}$ and 8 $\mathrm{kHz}$.

3. A "dip" or reduction in sensitivity characteristically occurs near $4 \mathrm{kHz}$.

These features distinguish the Old World monkeys from the prosimians on the one hand, and from the apes and man on the other. The former have a frequency range of hearing extending to $70 \mathrm{kHz}$ and are relatively less sensitive to frequencies below $8 \mathrm{kHz}$; man's upper frequency limit is about $20 \mathrm{kHz}$ and the chimpanzee's about $30 \mathrm{kHz}$ (Stebbins, '71). Thus the cercopithecine monkey may reflect a stage in the evolution of human hearing - a trend toward reduction in high frequency hearing and slightly enhanced sensitivity to the low and mid- range frequencies. The selective pressures in evolution may have played a role in the development of high frequency hearing which in turn produced more accurate sound localization in some of the mammals and in the nocturnal prosimians (Masterton, Heffner and Ravizza, '69). As Masterton has pointed out, the anthropoid primates with larger heads and greater interaural spacing no longer had to rely on high frequencies for accurate sound localization, but instead could make use of interaural time difference as a cue for localization.

The absolute threshold function for hearing (fig. 3) provides information on the minimum detectable energy levels of sound to which the monkey is capable of responding. Two other important characteristics of the auditory system concern its resolving power for acoustic frequency and amplitude (sound pressure). The experimental question refers to the minimal difference in frequency and intensity (sound pressure) to which the monkey can respond. In figure 4 , the frequency difference thresholds $(\Delta \mathrm{F})$ at $60 \mathrm{~dB} \mathrm{SL}$, i.e., $60 \mathrm{~dB}$ above the threshold of hearing, for two different species of Macaca are shown as a function of the standard frequency. The method of threshold determination is illustrated in 


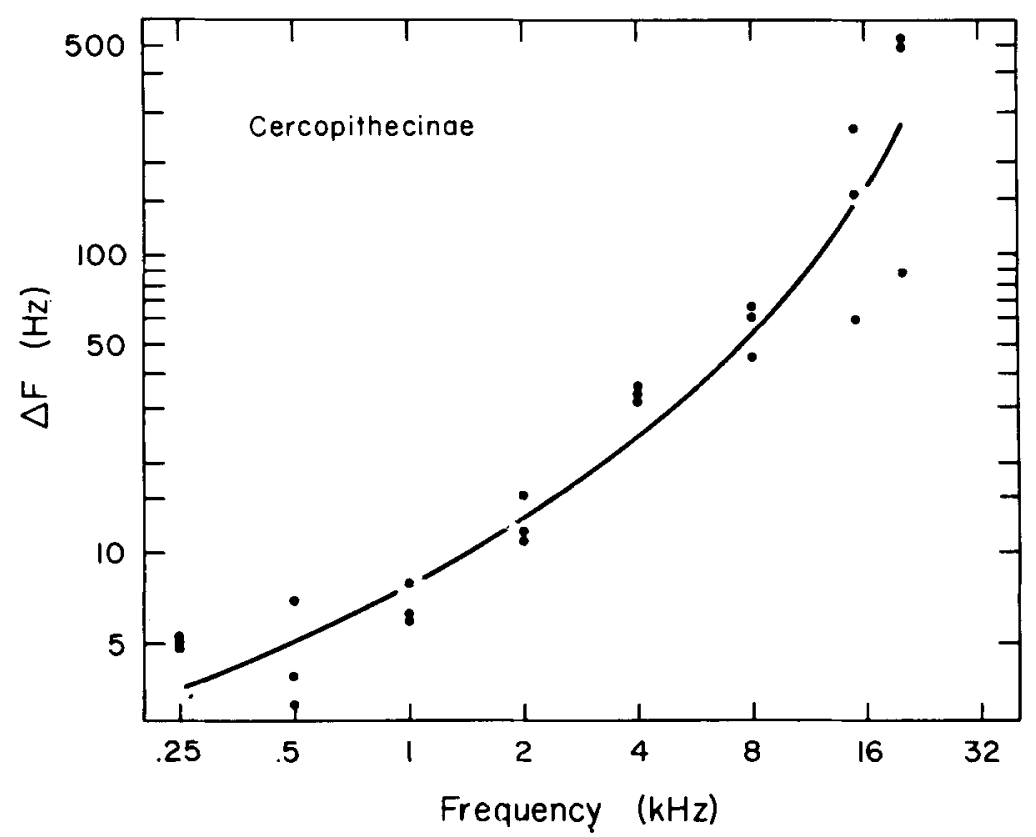

Fig. 4 Frequency difference threshold at $60 \mathrm{~dB}$ SL as a function of frequency for three subjects representing two species of macaque.

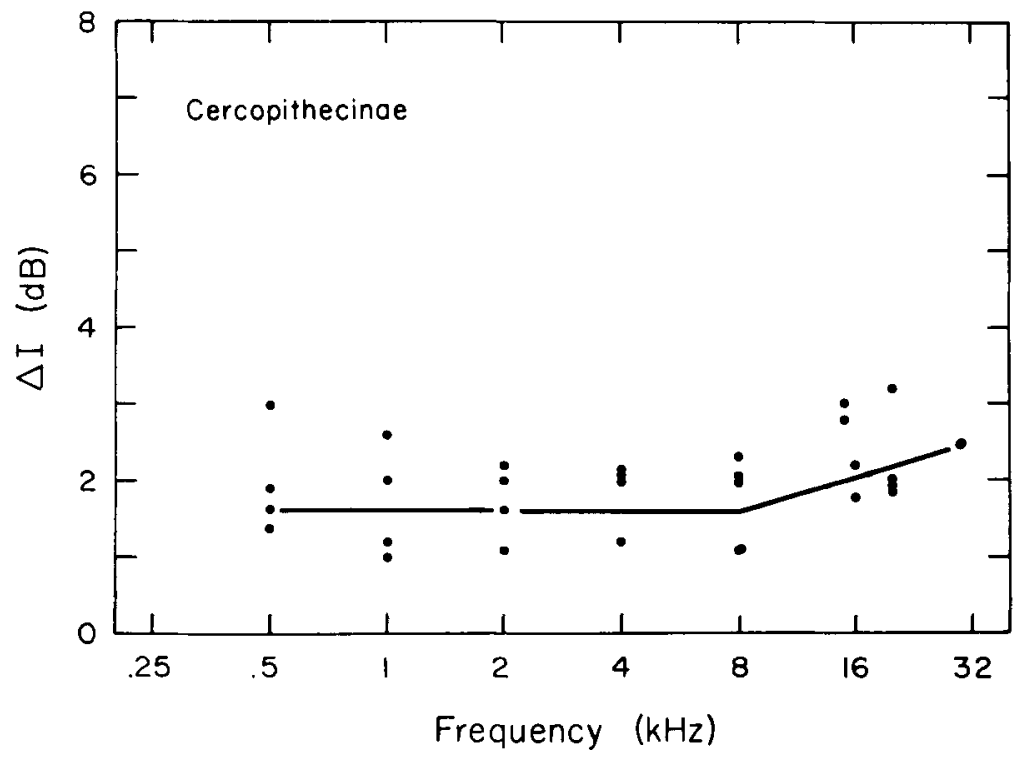

Fig. 5 Intensity difference threshold at $60 \mathrm{~dB}$ SL as a function of frequency for four subjects representing two genera of Cercopithecinae.

figure 2. For example, for a tone of $1 \mathrm{kHz}$, for man are about $2 \mathrm{~Hz}$ (at $500 \mathrm{~Hz}$ ) and the monkey's threshold of discriminability is slightly above $5 \mathrm{~Hz}$; at $8 \mathrm{kHz}$, it has increased to about $50 \mathrm{~Hz}$. Comparable values $20 \mathrm{~Hz}$ (at $8 \mathrm{kHz}$ ), respectively. The increase in $\Delta \mathrm{F}$ with frequency for monkey and man is characteristic; man's differential acuity 


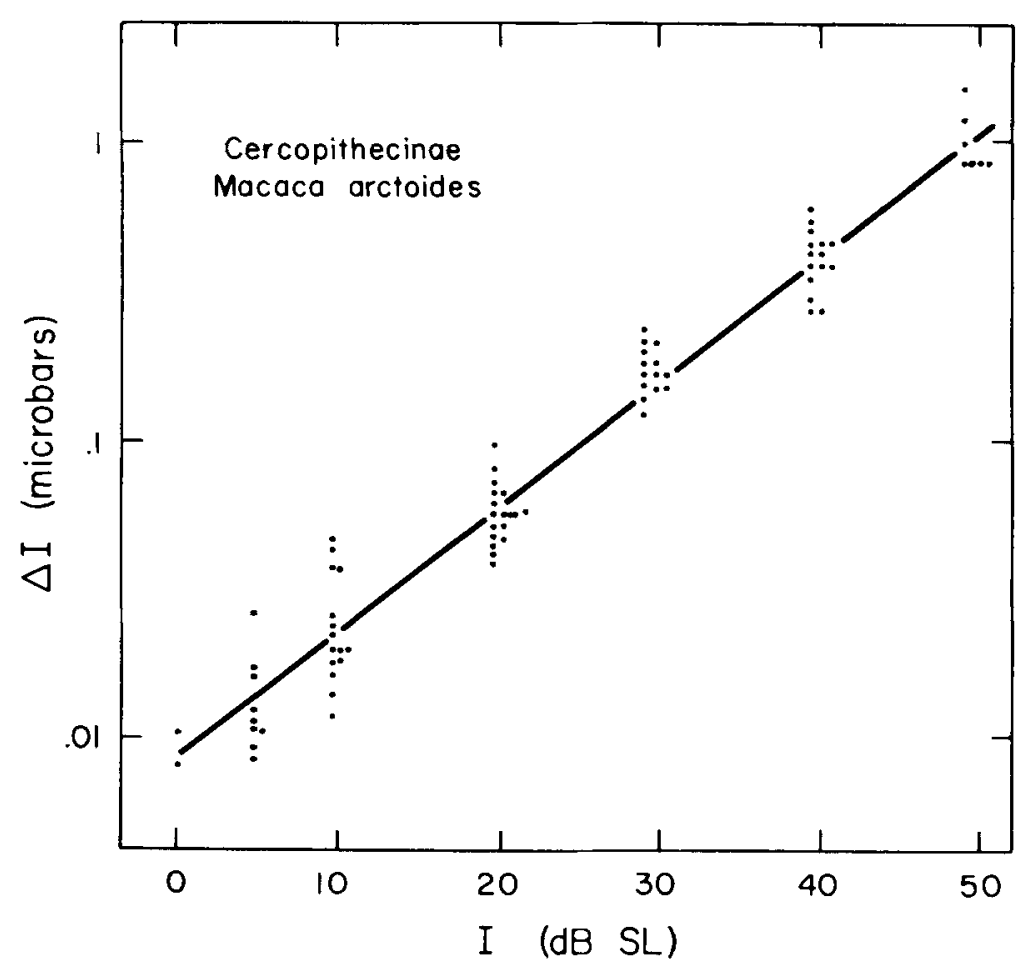

Fig. 6 Intensity difference threshold as a function of sensation level, i.e., of intensity above the threshold of hearing for one typical subject.

for frequency differences is slightly sharper (Licklider, '51).

In figure 5 , the intensity difference thresholds $(\Delta \mathrm{I})$ at $60 \mathrm{~dB}$ SL for two different species of Macaca and one of Papio are shown as a function of frequency. The difference threshold is relatively constant at about $2 \mathrm{~dB}$ across frequency with a slight increase suggested at the higher frequencies. The shape of the function for man is very similar with a lower difference threshold of less than $1 \mathrm{~dB}$. The intensity difference threshold increases as the intensity level at which it is determined is increased. These findings are shown for one macaque at several levels above the threshold of hearing in figure 6. For example, at $5 \mathrm{~dB}$ $\mathrm{SL}, \Delta \mathrm{I}=0.01 \mu$ bar; at $50 \mathrm{~dB} \mathrm{SL}, \Delta \mathrm{I}=1$ $\mu$ bar. The proportional relation obtains for man also (Licklider, '51).

\section{CONCLUSIONS}

The range of hearing for the cercopithecine monkey extends from below $60 \mathrm{~Hz}$ to about $40 \mathrm{kHz}$.
Maximum sensitivity is in the midrange at $1 \mathrm{kHz}$ and $8 \mathrm{kHz}$ with a region of reduced sensitivity between these values.

The difference threshold for frequency increases with frequency from a low of about $5 \mathrm{~Hz}$ at 250 and $500 \mathrm{~Hz}$ to a difference threshold as high as $500 \mathrm{~Hz}$ at 20 $\mathrm{kHz}$.

The difference threshold for sound pressure is constant at about $2 \mathrm{~dB}$ for all frequencies.

The difference threshold for sound pressure is proportional to the intensity above the threshold of hearing at which it is determined.

These basic functions obtain also for man with certain important differences: man's upper frequency limit is $20 \mathrm{kHz}$; his differential acuity for both frequency and intensity is slightly superior to that of the old World monkey.

\section{LITERATURE CITED}

Licklider, J. C. R. 1951 Basic correlates of the auditory stimulus. In: Handbook of Experi- 
mental Psychology. S. S. Stevens, ed. John Wiley \& Sons, Inc., New York, pp. 985-1039.

Masterton, B., H. Heffner and R. Ravizza 1969 The evolution of human hearing. J. Acoust. Soc. Amer., 45: 966-985.

Moody, D. B., W. C. Stebbins and J. M. Miller 1970 A primate restraint and handling system for auditory research. Behav. Res. Meth. \& Instru., 2: 180-182.

Napier, J. R., and P. H. Napier 1967 A Handbook of Living Primates. Acadernic Press, London.

Stebbins, W. C, 1970 Studies of hearing and hearing loss in the monkey. In: Animal Psycho- physics: The design and conduct of sensory experiments. W. C. Stebbins, ed. AppletonCentury-Crofts, New York, pp. 41-66.

1971 Hearing. In: Behavior of Nonhuman Primates. A. M. Schrier and F. Stollnitz, eds. Academic Press, New York. Vol. 3, pp. 159192.

Stebbins, W. C., and S. Coombs 1973 Chapt. 4. Behavioral assessment of ototoxicity in nonhuman primates. In: Proceedings of Conference on Behavioral Toxicology, B. Weiss and V. G. Laties, eds. Appleton-Century-Crofts, New York, in press. 